\title{
Simulation of Hypervelocity Impact on Massively Parallel Supercomputer*
}

\author{
H. Eliot Fang \\ Computational Physics and Mechanics Department \\ Sandia National Laboratories \\ Albuquerque, New Mexico, U. S. A.
}

\section{INTRODUCTION}

Hypervelocity impact studies are important for debris shield and armor/anti-armor research and development. Numerical simulations are frequently performed to complement experimental studies, and to evaluate code accuracy. Parametric computational studies involving material properties, geometry and impact velocity can be used to understand hypervelocity impact processes. These impact simulations normally need to address shock wave physics phenomena, material deformation and failure, and motion of debris particles. Detailed, three-dimensional calculations of such events have large memory and processing time requirements. At Sandia National Laboratories, many impact problems of interest require tens of millions of computational cells. Furthermore, even the inadequately resolved problems often require tens or hundred of Cray CPU hours to complete.

Recent numerical studies done by Grady and Kipp [1] at Sandia using the Eulerian shock wave physics code CTH [2] demonstrated very good agreement with many features of a copper sphere-on-steel plate oblique impact experiment, fully utilizing the compute power and memory of Sandia's Cray supercomputer. To satisfy requirements for more finelyresolved simulations in order to obtain a better understanding of the crater formation process and impact ejecta motion, the numerical work has been moved from the shared-memory Cray to a large, distributed-memory, massively parallel supercomputing system using PCTH [3-5], a parallel version of CTH.

The current work is a continuation of the studies in [1], but done on Sandia's Intel 1840processor Paragon X/PS parallel computer. With the great compute power and large memory provided by the Paragon, a highly detailed PCTH calculation has been completed for the copper sphere impacting steel plate experiment. Although the PCTH calculation used a mesh which is 4.5 times bigger than the original Cray setup, it finished in much less CPU time.

* This work was performed at Sandia National Laboratories supported by the U.S. Department of Energy under Contract DE-AC04-94AL85000.

DISTRIBUTION OF THIS DOCUMENT IS UNLIMITED 


\section{DISCLAIMER}

Portions of this document may be illegible in electronic image products. Images are produced from the best available original document. 


\section{THE COPPER SPHERE-ON-STEEL PLATE IMPACT EXPERIMENT}

Recently at Sandia, Grady and Kipp [1] performed a series of hypervelocity impact experiments to investigate the dynamic fragmentation characteristics of a high-strength steel under hypervelocity impacts, and to validate CTH for its capability of fragmentation prediction. These experiments used copper spheres to strike 4340 steel target plates at velocities between 3 and $5 \mathrm{~km} / \mathrm{s}$, and at both normal and oblique impact angles. Both the former and the current numerical studies focus on only one of the experiments, which has a copper sphere obliquely impacting on a stationary steel plate at 30.8 degree angle to the normal, with $4.52 \mathrm{~km} / \mathrm{s}$ impact velocity. The copper sphere was $6.355 \mathrm{~mm}$ in diameter and the steel plate was $5.6 \mathrm{~mm}$ thick. The initial impact geometry used in the current simulation is given in Figure 1. Figure 2 presents an experimental radiograph which was taken $11.6 \mu \mathrm{s}$ after impact. The slight asymmetry in the envelope of the behind-target ejecta debris, the size of the envelope behind the target plate, the hole dimension after impact, and the curvature of the back-splash debris as well as the splash angle are features that numerical studies intend to match.

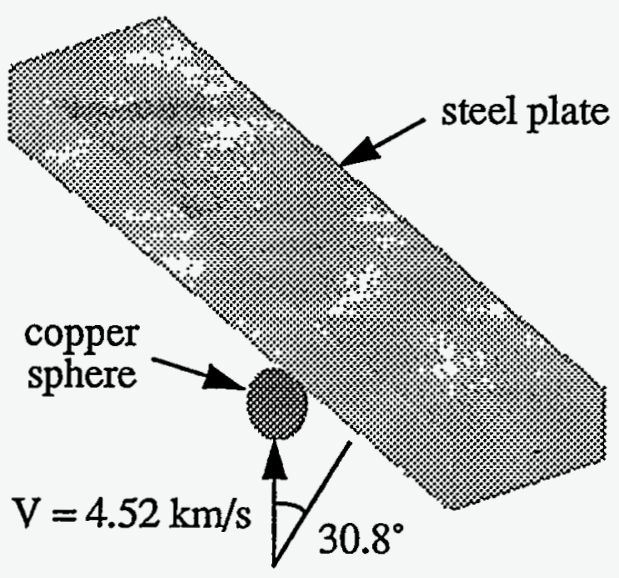

Figure 1. Initial impact Geometry.

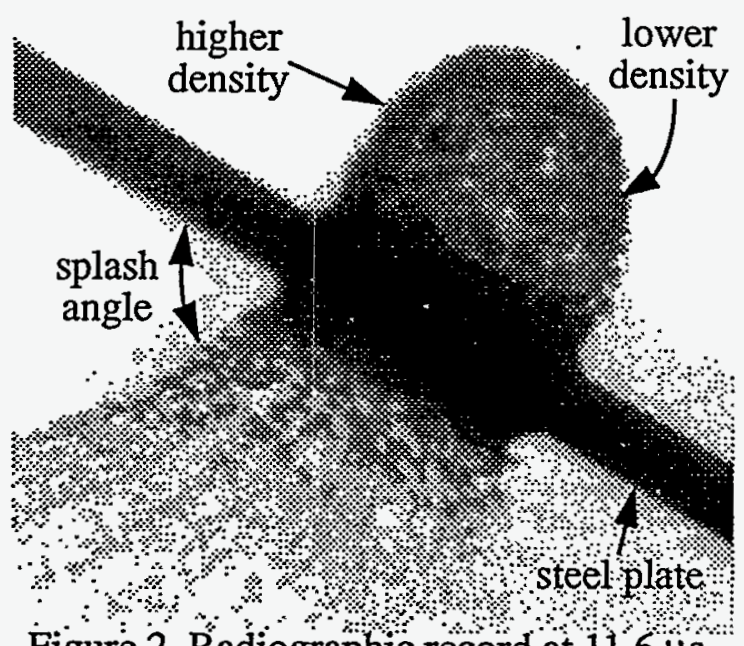

Figure 2. Radiographic record at $11.6 \mu$ s.

\section{SIMULATION TOOL --- MASSIVELY PARALLEL COMPUTING}

Massively parallel computing is a technology in which hundreds or thousands of processors work cooperatively to carry out computations beyond the capabilities of current vector supercomputers. Many computational techniques, including finite-difference descriptions for dynamic simulations, contain inherently parallel components, and hence can be solved by parallel algorithms. Each processor in a massively parallel computer is allocated a portion of a problem to solve, and works on its portion in parallel with the other processors. An additional cost is incurred in communicating results among processors, but it can in general be made a small fraction of the computational cost. This makes massively 
parallel computing feasible and potentially more powerful than vector supercomputing. In the following sections, the hardware and the application code used in the current simulation work are described briefly.

\subsection{HARDWARE --- INTEL PARAGON PARALLEL SUPERCOMPUTER}

Sandia's Intel Paragon X/PS L-140 parallel supercomputer is the main hardware used in the current work. The machine has 1840 computational nodes, each with an i860 XP RISC processor for computation and an additional i860 XP processor devoted to inter-node communication. The nodes are connected via a two-dimensional mesh communications topology. All coordination among nodes is performed via explicit message passing calls. Each node runs its own copy of the operating system. The $1860 \mathrm{XP}$ processor is rated at 75 Megaflop/s for double-precision (64-bit) floating point operations. Typical performance is four to eight Megaflop/s (double precision) per node for both Fortran and C. The 1840-node configuration has a total memory of approximately 38 Gigabytes and a peak theoretical speed of 138 Gigaflop/s. Table 1 summarizes the comparison between Sandia's Paragon and Cray Y-MP. All the performance speeds shown in Table 1 are peak values and rated with 64bit processing.

\begin{tabular}{|l|c|c|}
\hline & Paragon & Cray Y-MP \\
\hline \hline Number of Processors & 1840 & 8 \\
\hline Hardware Type & Parallel & Vector \\
\hline Processor Speed & $75 \mathrm{MFlop} / \mathrm{s}$ & $333 \mathrm{MFlop} / \mathrm{s}$ \\
\hline Full Machine Speed & $138 \mathrm{GFlop} / \mathrm{s}$ & $2.66 \mathrm{GFlop} / \mathrm{s}$ \\
\hline Memory Type & Distributed & Shared \\
\hline Memory Arrangement & $32 \mathrm{MB} /$ node for 512 nodes & $64 \mathrm{MWds}$ RAM (512 MB) \\
& $16 \mathrm{MB} /$ node for 1328 nodes & $200 \mathrm{MWds}$ SSD (1600 MB) \\
\hline Total Memory & $37.632 \mathrm{~GB}$ & $2.112 \mathrm{~GB}$ \\
\hline
\end{tabular}

Table 1: Comparison between Sandia's Paragon and Cray Y-MP.

\subsection{APPLICATION CODE --- PCTH (PARALLEL CTH)}

Sandia scientists have developed a parallel version of the CTH shock physics code [2]. The parallel code, called PCTH, is a multidimensional, multimaterial, finite-difference shock physics code which models large deformation and shocks, and the multiphase behavior, strength, and fracture of materials. Its algorithms are implemented using modern object-oriented numerics techniques in the $\mathrm{C}++$ programming language. The code is designed to be easily portable to message-passing parallel computers, and currently runs on the nCUBE2, the Intel iPSC/860, the Paragon, and networks of workstations. Note that CTH is written in Fortran 77 and runs on many computing platforms. 
In PCTH, the equations governing the conservation of mass, momentum and energy are integrated explicitly in time using a two-step Eulerian scheme. The first step is a Lagrangian step in which the computational cells distort to follow the material motion, using an algorithm which is second-order accurate in space and time. The second step is an advection or remapping step in which the distorted cells are mapped back to the Eulerian mesh using a second-order van Leer advection scheme. Two interface trackers are available for threedimensional interface reconstruction: SLIC (Simple Line Interface Calculation) [6], a firstorder accurate scheme; and SMYRA (Sandia Modified Young's interface Reconstruction Algorithm) [7], a second-order accurate scheme.

\section{SIMULATION MODELS}

The oblique impact experiment of copper sphere-on-steel plate was modeled using a three-dimensional mesh with $27,525,120$ cubical cells of dimension $0.15625 \mathrm{~mm}$ cubes. Such a resolution allows 41 cells across the diameter of the copper sphere and 36 cells across the thickness of the steel plate. A symmetry plane, namely the vertical plane through the axis of impact, was used to reduce the number of computational cells. SLIC was selected as the interface tracker for interface reconstruction. The copper sphere and steel plate were modeled as linearly-elastic, perfectly-plastic materials. Fracture was simulated by the maximum tensile stress criterion/void insertion model. A simple Mie-Grüneisen equation of state [8] without material phase transformation was employed for both the copper and steel. Table 2 lists the material parameters used in the PCTH calculation.

\begin{tabular}{|l|c|c|}
\hline \multicolumn{1}{|c|}{ Property } & Copper & Steel \\
\hline \hline Density $\left(\mathrm{kg} / \mathrm{m}^{3}\right)$ & 8930 & 7850 \\
\hline Bulk Sound Speed (m/s) & 3940 & 3574 \\
\hline $\begin{array}{l}\text { Linear Shock Velocity - } \\
\text { Particle Velocity Slope }\end{array}$ & 1.489 & 1.920 \\
\hline Grüneisen Coefficient & 1.99 & 1.69 \\
\hline Yield Strength (GPa) & 0.7 & 2.0 \\
\hline Poissons Ratio & 0.3506 & 0.2897 \\
\hline Fracture Stress (GPa) & 0.3 & 1.2 \\
\hline
\end{tabular}

Table 2: Material parameters.

\section{RESULTS AND DISCUSSION}

A sequence of images generated from the PCTH calculation is presented in Figure 3 to show the interaction between the sphere and plate at the symmetry plane. Figure 4 provides 
a plot of the PCTH result which shows the interior of the envelope behind the target plate, with a view through the symmetry plane. A plot of the CTH result is given in Figure 5 for comparison. In Figures 4 and 5, the dark color represents copper and the light color is steel. The oblique impact angle is clearly reflected in the asymmetry of material mass in the debris cloud formed behind the target. The material distribution calculated by PCTH agrees with what can be observed from the experimental radiograph (Figure 2) very well. The deformation and material distribution of the 27.5 million cell PCTH calculation appears to be very similar to the 6 million cell CTH calculation: both have captured the essential features. In both cases the appearance of fragments is characteristic of the particular resolution of the problem.

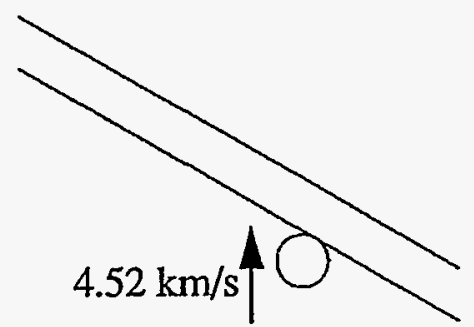

$0 \mu \mathrm{s}$

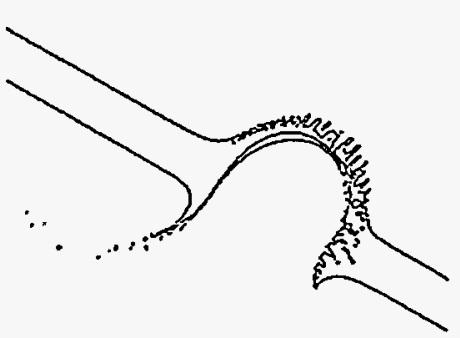

$6 \mu \mathrm{s}$

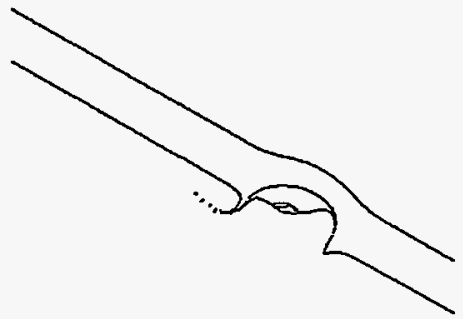

$2 \mu \mathrm{s}$

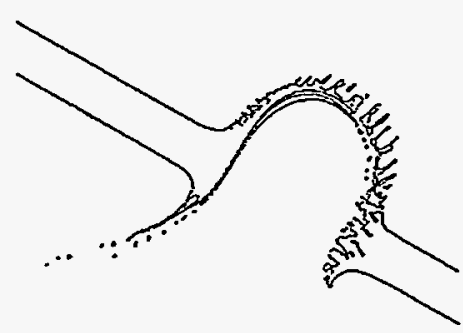

$8 \mu \mathrm{s}$

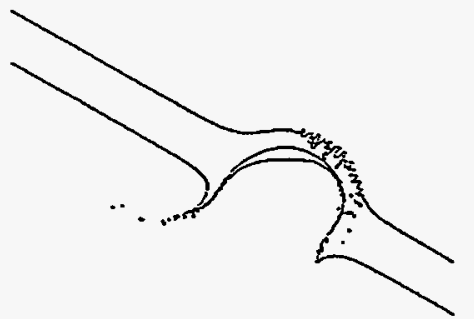

$4 \mu s$

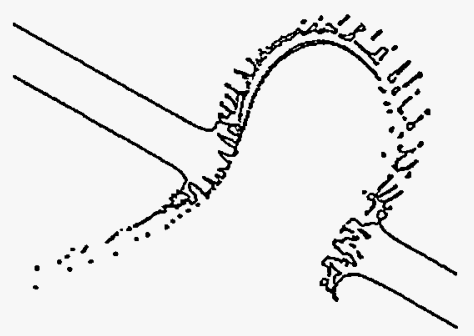

$11.6 \mu \mathrm{s}$

Figure 3. Calculated material interaction on the plane of symmetry at selected times.

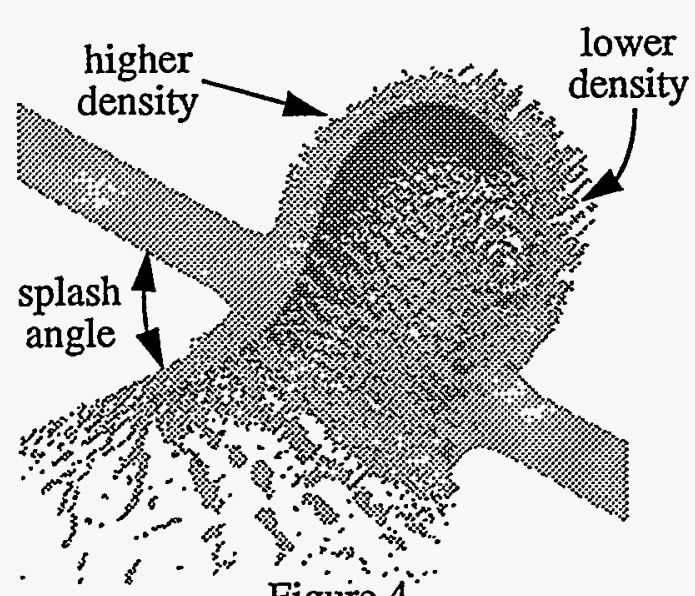

Figure 4 :

PCTH result (27.5 million cells) at $11.6 \mu$ s.

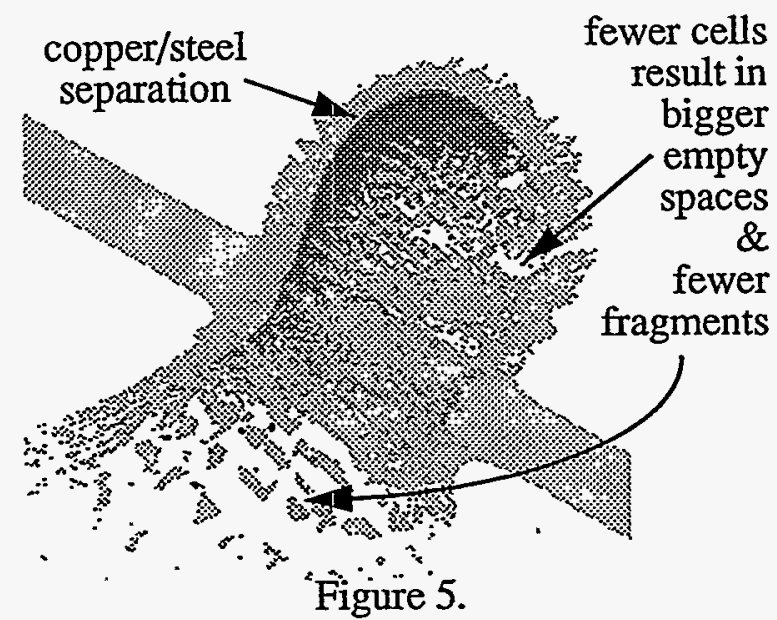

CTH result ( 6 million cells) at $11.6 \mu \mathrm{s}$. 
Comparison of the PCTH calculation on the Paragon with the previous CTH calculation and the experimental radiograph provides support for the validation of this newly developed PCTH shock wave physics code for hypervelocity impact simulations. In addition, the current work demonstrates that massively parallel computing can provide rapid, highly detailed, three-dimensional simulations of real-world problems. CTH took about $100 \mathrm{CPU}$ hours on the Cray to finish the 6 million cell calculation, whereas PCTH used only 21.5 hours to complete the 27.5 million cell calculation. That provided about a $50 \%$ improvement in spatial resolution. With the fast performance and resolution improvement from PCTH/ Paragon, parameter studies become feasible to examine the effects of interface trackers and material models and equations of state on the projectile/target response. For example, while the increase in resolution did not alter the separation observed between the copper and the steel at $11.6 \mathrm{~ms}$ (See Figure 5 for the location.), inclusion of an equation of state with melt or applying a different interface tracker may influence this response. Computational studies to investigate the influence of material parameters and numerical schemes are now feasible and affordable with the rapid turnaround provided by PCTH calculations on the Paragon.

\section{ACKNOWLEDGEMENTS}

The author gratefully acknowledge Dr. Marlin E. Kipp of Sandia National Laboratories for his many helpful suggestions.

\section{REFERENCES}

[1] D. E. Grady and M. E. Kipp, Experimental and Computational Simulation of the High Velocity Impact of Copper Spheres on Steel Plates, Int. J. Impact Engineering, Vol. 15, No. 5 (1994), pp. 645-660.

[2] J. M. McGlaun, S. L. Thompson and M. G. Elrick, CTH: A Three-Dimensional Shock Wave Physics Code, Int. J. Impact Engineering, Vol. 10 (1990), pp. 351-360.

[3] A. C. Robinson, A. L. Ames, H. E. Fang, D. Pavlakos, C. T. Vaughan and P. Campbell, Massively Parallel Computing, $\mathrm{C}++$ and Hydrocode Algorithms, Proc. of the Computing in Civil Engineering, 8th Conf. in conjunction with A/E/C Systems (1992), pp. 519-526. . . . [4] H. E. Fang and A. C. Robinson, 3-D Massively Parallel Impact Simulations Using PCTH, Proc. of the 1993 Summer Computer Simulation Conference (1993), pp. 385-390.

[5] J. M. McGlaun, Parallel Solid Mechanics Codes at Sandia National Laboratories, Proc. of the 1994 International Mechanical Engineering Congress and Exposition, High Performance Computing in Computational Dynamics (1994), pp. 89-94.

[6] W. F. Noh and P. Woodward, SLIC (Simple Line Interface Calculation), Proc. of the 5th Int. Conf. on Numerical Methods in Fluid Dynamics (1976), pp. 330-340.

[7] R. L. Bell and E. S. Hertel, Jr., An Improved Interface Reconstruction Algorithm for Eulerian Codes, Sandia National Laboratories Report SAND92-1716 (1992).

[8] G. I. Kerley, CTH Reference Manual: The Equation of State Package, Sandia National Laboratories Report SAND91-0344 (1991). 


\section{DISCLAIMER}

This report was prepared as an account of work sponsored by an agency of the United States Government. Neither the United States Government nor any agency thereof, nor any of their employees, makes any warranty, express or implied, or assumes any legal liability or responsibility for the accuracy, completeness, or usefulness of any information, apparatus, product, or process disclosed, or represents that its use would not infringe privately owned rights. Reference herein to any specific commercial product, process, or service by trade name, trademark, manufacturer, or otherwise does not necessarily constitute or imply its endorsement, recommendation, or favoring by the United States Government or any agency thereof. The views and opinions of authors expressed herein do not necessarily state or reflect those of the United States Government or any agency thereof. 